\title{
A Review of Warfarin Dosing and Monitoring
}

\author{
KM Arif ${ }^{1}$ MA Rahman ${ }^{2}$
}

\begin{abstract}
:
The anticoagulant drug warfarin is a vitamin $\mathrm{K}$ antagonist, coumarin derivative which inhibits the synthesis of clotting factors II, VII, IX, and X, as well as the naturally occurring endogenous anticoagulant proteins C and S. Warfarin is still considered the mainstay of oral anticoagulant treatment, it is a difficult drug to manage due to its narrow therapeutic index. An inappropriate management of patients can lead to subtherapeutic or supratherapeutic levels, increasing the risk of thromboembolic episodes or hemorrhagic episodes, respectively. Common indications for the use of warfarin include stroke prevention in atrial fibrillation, preventing thrombus formation in patients with heart valves and treatment of venous thromboembolism. When warfarin therapy is initiated for venous thromboembolism, it should be given the first day, along with a heparin product or fondaparinux. The heparin product or fondaparinux should be continued for at least five days and until the patient's international normalized ratio is at least 2.0 for two consecutive days. The international normalized ratio goal and duration of treatment with warfarin vary depending on indication and risk. Warfarin therapy should be stopped five days before major surgery and restarted 12 to 24 hours postoperatively. Bridging with low-molecular-weight heparin or other agents is based on balancing the risk of throm boembolism with the risk of bleeding.
\end{abstract}

Key words: Warfarin, Anticoagulants, Antithrombotics.

\section{Introduction:}

Warfarin is a vitamin $\mathrm{K}$ antagonist (VKA), coumarin derivative. Chemist Paul Link and his student Harold Campbell, working at the University of Wisconsin, found potent coumarin-based anticoagulants for use as rodent poisons, resulting in warfarin in 1948. The name warfarin stems from the acronym WARF, for Wisconsin Alumni Research Foundation + the ending-arin indicating its link with coumarin.

\section{Pharmacokinetics and Pharmacodynamics:}

The anticoagulant drug warfarin is a vitamin $\mathrm{K}$ antagonist (VKA), coumarin derivative, formed by the racemic mixture of two optically active isomers known by Rectus (R) and Sinister (S) enantiomers in equal proportion, being the $\mathrm{S}$-warfarin five times more potent. It is readily absorbed from GIT, highly plasma protein bound; onset of action is 36-48 hours after

1. Dr. Khan Md. Arif, MBBS, FCPS (Medicine), Assistant Professor, Department of Medicine, Faridpur Medical College, Faridpur.

2. Dr. Mohammad Ashiqur Rahman, MBBS, Registrar, Department of Medicine, Faridpur Medical College Hospital, Faridpur.

Address of correspondence :

Dr. Khan Md. Arif, MBBS, FCPS (Medicine), Assistant Professor, Department of Medicine, Faridpur Medical College, Faridpur. Mobile: +88-01711-986724, E-mail: ingestion. Plasma half life is $20-40$ hours (36 hours average), duration of action: 2-6 days. It is metabolized in liver (microsomal enzymes depended) \& excreted through urine, bile.

Warfarin acts by interfering in the interconversion of cyclic 2,3 vitamin $\mathrm{K}$ epoxide. The vitamin $\mathrm{K}$ epoxide reductase is inhibited by therapeutic doses of warfarin inhibiting thus the synthesis of vitamin K-dependent factors, leading to inhibition of $\mathrm{Y}$-carboxylation of clotting factors II, VII, IV and X, and the anticoagulant proteins $\mathrm{C}$ and $\mathrm{S}$. Effects on prothrombin time are produced in 24 to 36 hours after the initial dose and reach the maximum plasmatic concentration in 36 to 48 hours, maintained for 48 hours or more after discontinuance of dosing.

\section{Clinical Management:}

Although warfarin is still considered the mainstay of oral anticoagulant treatment, it is a difficult drug to manage due to its narrow therapeutic index, metabolism that is affected by many factors and numerous drug interactions. An inappropriate management of patients can lead to subtherapeutic or supratherapeutic levels, increasing the risk of 
thromboembolic episodes or hemorrhagic episodes, respectively. Common indications for the use of warfarin include stroke prevention in atrial fibrillation, preventing thrombus formation in patients with heart valves and treatment of venous thromboembolism.
Things to be Considered when Starting Warfarin

* Ensure that patient doesn't have any of these absolute contraindication for warfarin

* Weigh risk of clotting with risk of bleeding

* Select appropriate target INR range

* Select appropriate treatment duration

* Select appropriate starting dose

\section{Table I: Warfarin Target INR Range and Length of Treatment}

\begin{tabular}{|c|c|c|c|}
\hline $\begin{array}{l}\text { Indication } \\
\text { DVT and } \mathrm{PE}^{1}\end{array}$ & Target & Duration and additional information INR & $\begin{array}{l}\text { Grade of } \\
\text { Recommendation }\end{array}$ \\
\hline $\begin{array}{l}\text { PE or DVT of leg provoked by } \\
\text { surgery or } \\
\text { transient/reversible risk } \\
\text { factor }\end{array}$ & $2-3$ & 3 months & $\begin{array}{l}\text { 1B (Strong } \\
\text { recommendation, } \\
\text { moderate quality } \\
\text { evidence) }\end{array}$ \\
\hline $\begin{array}{l}\text { PE or DVT of leg unprovoked } \\
\text { by surgery or } \\
\text { transient/reversible risk } \\
\text { factor }\end{array}$ & $2-3$ & $\begin{array}{l}\text { At least } 3 \text { months (over shorter period), then } \\
\text { evaluate for risk -benefit of extended therapy. } \\
\text { In patients with a first VTE that is an unprovoked } \\
\text { proximal DVT of the leg or PE and who have a low } \\
\text { or moderate bleeding risk, use extended } \\
\text { anticoagulant therapy (no scheduled stop date) } \\
\text { over } 3 \text { months of therapy (Grade } 2 \mathrm{~B} \text { ). If high } \\
\text { bleeding risk, use } 3 \text { months of anticoagulant } \\
\text { therapy over extended therapy (no scheduled stop } \\
\text { date) (Grade 1B). }\end{array}$ & $\begin{array}{l}\text { 1B (Strong } \\
\text { recommendation, } \\
\text { moderate quality } \\
\text { evidence) }\end{array}$ \\
\hline $\begin{array}{l}\text { PE or DVT of leg in patients } \\
\text { with active cancer }\end{array}$ & $2-3$ & $\begin{array}{l}\text { Extended ( }>3 \text { months) } \\
\text { Suggest use of LMWH over warfarin in PE or DVT } \\
\text { of leg }\end{array}$ & $\begin{array}{l}\text { 1B ( } 2 \mathrm{~B} \text { if high-risk fo } \\
\text { bleed) } \\
\text { 2B (Weak } \\
\text { recommendation) }\end{array}$ \\
\hline \multicolumn{4}{|c|}{ Non valvular atrial fibrillation and/or flutter ${ }^{2}$} \\
\hline $\begin{array}{l}\text { Low risk } \\
\text { (CHA2DS2-VASc }=0)\end{array}$ & N/A & Reasonable to omit antithrombotic therapy & $\begin{array}{l}2 \mathrm{~A} \text { (Weak } \\
\text { recommendation, } \\
\text { high quality } \\
\text { evidence) }\end{array}$ \\
\hline $\begin{array}{l}\text { Intermediate risk (CHA2DS2- } \\
\text { VASC }=1 \text { ) }\end{array}$ & $2-3$ & $\begin{array}{l}\text { No antithrombotic therapy or long -term treatment } \\
\text { with an oral anticoagulant or aspirin may be } \\
\text { considered }\end{array}$ & $\begin{array}{l}2 \mathrm{~B} \text { (Weak } \\
\text { recommendation, } \\
\text { moderate quality } \\
\text { evidence) }\end{array}$ \\
\hline $\begin{array}{l}\text { High risk } \\
\text { (CHA2DS2-VASc > 2) }\end{array}$ & $2-3$ & Long-term & $\begin{array}{l}1 \mathrm{~A} \text { (Strong } \\
\text { recommendation, } \\
\text { high quality } \\
\text { evidence) }\end{array}$ \\
\hline Valvular Disease $^{3}$ & $2-3$ & $\begin{array}{l}\text { At least } 3 \text { weeks prior to and at least } 4 \text { weeks after } \\
\text { regardless of CHA2DS2 -VASc score or method of } \\
\text { cardioversion. }\end{array}$ & $\begin{array}{l}\text { 1B (Strong } \\
\text { recommendation, } \\
\text { moderate quality } \\
\text { evidence) }\end{array}$ \\
\hline
\end{tabular}


Table II: Warfarin Initiation Nomogram (5mg starting dose, target INR range $2-3)^{5}$

\begin{tabular}{|c|c|c|}
\hline & INR & Dose \\
\hline DAY 1 & & $5 \mathrm{mg}$ \\
\hline \multirow[t]{4}{*}{ DAY 2} & $<1.5$ & $5 \mathrm{mg}$ \\
\hline & $1.5-1.9$ & $2.5 \mathrm{mg}$ \\
\hline & $2.0-2.5$ & $1-2.5 \mathrm{mg}$ \\
\hline & $>2.5$ & $0 \mathrm{mg}$ \\
\hline \multirow[t]{4}{*}{ DAY 3} & $<1.5$ & $5-10 \mathrm{mg}$ \\
\hline & $1.5-1.9$ & $2.5-5 \mathrm{mg}$ \\
\hline & $2.0-3.0$ & $0-2.5 \mathrm{mg}$ \\
\hline & $>3.0$ & $0 \mathrm{mg}$ \\
\hline \multirow[t]{4}{*}{ DAY 4} & $<1.5$ & $10 \mathrm{mg}$ \\
\hline & $1.5-1.9$ & $5-7.5 \mathrm{mg}$ \\
\hline & $2.0-3.0$ & $0-5 \mathrm{mg}$ \\
\hline & $>3.0$ & 0 \\
\hline \multirow[t]{4}{*}{ DAY 5} & $<1.5$ & $10 \mathrm{mg}$ \\
\hline & $1.5-1.9$ & $7.5-10 \mathrm{mg}$ \\
\hline & $2.0-3.0$ & $0-5 \mathrm{mg}$ \\
\hline & $>3.0$ & 0 \\
\hline \multirow[t]{4}{*}{ DAY 6} & $<1.5$ & 7.5 - $12.5 \mathrm{mg}$ \\
\hline & $1.5-1.9$ & $5-10 \mathrm{mg}$ \\
\hline & $2.0-3.0$ & $0-7.5 \mathrm{mg}$ \\
\hline & $>3.0$ & 0 \\
\hline
\end{tabular}

Table III: Warfarin dosing adjustment guidelines; for long-term therapy ${ }^{6}$

\begin{tabular}{|c|c|c|c|}
\hline $\begin{array}{l}\text { PATIENT } \\
\text { INR }\end{array}$ & GOAL $=1.5-2.0 \mathrm{INR}$ & $\begin{array}{l}\text { GOAL= 2.0-3.0 } \\
\text { INR }\end{array}$ & $\begin{array}{l}\text { GOAL }=2.5-3 \cdot 5 \\
\text { INR }\end{array}$ \\
\hline$<1.5$ & Increase $5-10 \%$ & Increase $5-20 \%$ & Increase $15-20 \%$ \\
\hline $1.5-2.0$ & Therapeutic & Increase $5-10 \%$ & Increase $5-20 \%$ \\
\hline $2.0-2.5$ & Decrease $5-10 \%$ & Therapeutic & Increase $5-10 \%$ \\
\hline $2.5-3.0$ & Decrease $5-15 \%$ & Therapeutic & Therapeutic \\
\hline $3.0-3.5$ & $\begin{array}{l}\text { (May Hold);Decrease } \\
10-20 \%\end{array}$ & $\begin{array}{l}\text { Decrease } 5- \\
10 \% / \text { stay same } \\
\text { if }>3.0\end{array}$ & Therapeutic \\
\hline $3.5-4.0$ & $\begin{array}{l}\text { Hold Dose; Decrease } \\
20-50 \%\end{array}$ & $\begin{array}{l}\text { (May } \\
\text { Hold);Decrease 5- } \\
10 \%\end{array}$ & $\begin{array}{l}\text { Decrease } 5^{-} \\
10 \% / \text { stay same if } \\
>3.5\end{array}$ \\
\hline $4 \cdot 0-4 \cdot 5$ & $\begin{array}{l}\text { Hold 2- } \\
3 \text { days; Decrease 20- } \\
50 \%\end{array}$ & $\begin{array}{l}\text { Hold } 1- \\
\text { 2days;Decrease } \\
10-20 \%\end{array}$ & $\begin{array}{l}\text { (May } \\
\text { Hold);Decrease 5- } \\
15 \%\end{array}$ \\
\hline
\end{tabular}

Drug Interaction ${ }^{7}$

* Potentiation of Drug Effect (Increased INR)

*Acetaminophen, Allopurinol, Amiodarone, Amoxicillin, Aspirin, Azithromycin, Bactrim(TMP-SMX), Cimetidine, Ciprofloxacin, Citalopram, Clarithromycin, Clopidogrel,
Cotrimoxazole, Diltiazem, Entacapone, Erythromycin, Fenofibrate, Fish Oil, Fluconazole, Fluvastatin, Gemcitabine, Gemfibrozil, Levofloxacin, Lovastatin, Metronidazole, Miconazole (Suppository and Gel), Omeprazole, Propafenone, Propanolol, Simvastatin, SSRI's, Tamoxifen, Tetracycline, Tramadol.

\section{* Inhibition of Drug Effect (Decreased INR)}

* Barbiturates, Bosentan, Carbamazepine, Cigarette Smoking, Chlordiazepoxide, Ginseng, Griseofulvin, Mercaptopurine, Multivitamin Supplement,Nafcillin, Phenobarbital, Ribavarin, Rifampin, Secobarbital, St. John's wort, Phenytoin.

\section{* Interaction with natural products and foods ${ }^{7}$}

Liver, Mustard greens, Soybean, Peas, Cauliflower, Pickles, Lettuce, Mayonnaise, Green beans,Cabbage, Spinach, Cole slaw, Broccoli, Brussels sprouts, Cranberry,Vit E, Fish oil,Sea weed, Soy Garlic, Green tea, Ginger, Glucosamine, Asparagus, Chondroiton, Avocado.

\section{Combination warfarin and antiplatelet therapy ${ }^{8}$ \\ Patients on antiplatelet therapy who develop an indication for warfarin}

* Patients receiving an anti-platelet agent as primary prophylaxis for CVD on developing an indication for warfarin, should stop their antiplatelet agent (1B, Strong recommendation, moderate quality evidence).

* Patients with peripheral artery disease or previous ischaemic stroke on antiplatelet therapy, should stop this agent if warfarin is commenced (1B, Strong recommendation, moderate quality evidence).

* Patients on aspirin or clopidogrel as secondary prophylaxis with stable ischaemic heart disease (often defined as $>12$ months following acute myocardial infarction), should stop their antiplatelet agent while being treated with warfarin (2B, Weak recommendation, moderate quality evidence).

* Patients on a single antiplatelet agent $<12$ months following an ACS, who require to start warfarin therapy, should continue aspirin therapy until 12 months post ACS, unless they are regarded as having a high bleeding risk (2B, Weak recommendation, moderate quality evidence).

* Patients on aspirin and clopidogrel, following an ACS or stent placement, who develop an indication 
for warfarin should be carefully assessed for bleeding risk and discussed with their cardiologist, with a view to introducing warfarin and minimizing the duration of triple therapy (2C, Weak recommendation, low quality evidence).

* When combined warfarin and single antiplatelet agent are indicated, consideration should be given to use of aspirin given the higher bleeding risk associated with clopidogrel (2C, Weak recommendation, low quality evidence).

\section{Patients on warfarin who develop an indication for antiplatelet agents}

* Patients requiring a coronary artery stent, should be considered for bare metal stent (rather than drugeluting stent) which would only necessitate triple therapy for 4 weeks, followed by aspirin and warfarin to 12 months (2B, Weak recommendation).

* Patients who do not undergo PCI should be considered for 4 weeks triple therapy, after which clopidogrel should be stopped, and aspirin continued for a further 11 months (2C, Weak recommendation, low quality evidence).

\section{Table IV: Guidelines for the management of} supratherapeutic INR

\begin{tabular}{|c|c|}
\hline $\begin{array}{l}\text { INR }>3<6 \text { (target INR } \\
2.5)\end{array}$ & Reduce warfarin dose/stop \\
\hline $\begin{array}{l}\text { INR }>4<6 \text { (target INR } \\
3.5)\end{array}$ & $\begin{array}{l}\text { (1) Reduce warfarin dose/omit. } \\
\text { (2) Restart when INR }<5.0\end{array}$ \\
\hline $\begin{array}{l}\text { INR }>6<8 \text { (no/minor } \\
\text { bleeding) }\end{array}$ & $\begin{array}{l}\text { (1) Stop warfarin } \\
\text { (2) Restart when INR }<5.0\end{array}$ \\
\hline $\begin{array}{l}\text { INR }>8 \text { (no/minor } \\
\text { bleeding) }\end{array}$ & $\begin{array}{l}\text { (1) Stop warfarin } \\
\text { (2) Restart warfarin when INR }<5.0 \\
\text { (3) If other risk factors for bleeding give } 0.5-2.5 \mathrm{mg} \text { of Vit } \\
\text { K(oral) }\end{array}$ \\
\hline Majorbleeding & $\begin{array}{l}\text { (1) Stop warfarin } \\
\text { (2)Give PCC } 50 \mathrm{U} / \mathrm{Kg}\left(\mathrm{FFP}_{15} \mathrm{ml} / \mathrm{Kg} \text {, if concentrate not }\right. \\
\text { available) } \\
\text { (3)Give } 5 \text {-10mg Vit K( oral/IV) }\end{array}$ \\
\hline Unexpected bleeding & Investigate the possibility of local anatomical cause \\
\hline
\end{tabular}

( $\mathrm{PCC}=$ Prothronbin Complex Concentrate, FFP=Fresh Frozen Plasma)

\section{Conclusion:}

Since warfarin has a narrow therapeutic window and has been associated with many drug-drug and drugfood interactions, patient counseling is crucial. The evaluation revealed this as an area for improvement because opportunities for patient education were not always optimized. Initiation and management of warfarin therapy is often difficult.Guidelines have been developed to assist the clinician in determining target ranges for therapeutic success. In addition, strategies for rapid anticoagulation and management of supratherapeutic INR values are also described in the literature. Daily practice using these guidelines should make management of patients easier when warfarin therapy is required.

\section{References :}

1. Kearon C, AklEA, Ornelas J, Blaivas A, Jimenez D, Bounameaux $\mathrm{H}$, et al. Antithrombotic Therapy for VTE Disease: CHEST Guideline and Expert Panel Report. Chest 2016; 149(2):315-52.

2. January CT, Wann LS, Alpert JS, Calkins H, Cigarroa JE, Cleveland JC Jr, et al. 2014 AHA/ACC/HRS guideline for the management of patients with atrial fibrillation: a report of the American College of Cardiology/American Heart Association Task Force on Practice Guidelines and the Heart Rhythm Society. J Am Coll Cardiol. 2014 Dec 2; 64(21):e1-76.

3. Nishimura RA, Otto CM, Bonow RO, Carabello BA, Erwin JP $3^{\text {rd }}$, Guyton RA, et al. 2014 AHA/ACC Guideline for the Management of Patients With Valvular Heart Disease: executive summary: a report of the American College of Cardiology/American Heart Association Task Force on Practice Guidelines. Circulation 2014; 129(23):2440-92.

4. Falck-Ytter Y, Francis CW, Johanson NA, Curley C, Dahl OE, Schulman S, et al. Prevention of VTE in orthopedic surgery patients: Antithrombotic Therapy and Prevention of Thrombosis, $9^{\text {th }}$ ed: American College of Chest Physicians Evidence-Based Clinical Practice Guidelines. Chest. 2012 Feb; 141(2 Suppl):e278S-e325S.

5. Harrison L, Johnston M, Massicotte MP, Crowther M, Moffat K, Hirsh J. Comparison of 5-mg and 10-mg loading doses in initiation of warfarin therapy. Ann Intern Med. 1997 Jan 15; 126(2):133-6

6. Kovacs MJ, Rodger M, Anderson DR, Morrow B, Kells G, Kovacs $\mathrm{J}$, et al. Comparison of $10-\mathrm{mg}$ and $5-\mathrm{mg}$ warfarin initiation nomograms together with low-molecular-weight heparin for outpatient treatment of acute venous thromboembolism. A randomized, double-blind, controlled trial. Ann Intern Med. 2003 May 6;138(9):714-9.

7. Holbrook AM, Pereira JA, Labiris R, McDonald H, Douketis JD, Crowther M, et al. Systematic overview of warfarin and its drug and food interactions. Arch Intern Med. 2005; 165:1095-106.

8. Keeling D, Baglin T, Tait C, Watson H, Perry D, Baglin C, et al. British Committee for Standards in Haematology.Guidelines on oral anticoagulation with warfarin - fourth edition. Br J Haematol. 2011 Aug; 154(3):311-24. 